\title{
The Analysis of National Sustainable Development Capability
}

\author{
Weichen Yang \\ School of Electrical and Electronic Engineering, North China Electric Power University, Baoding, \\ Hebei 071003, China. \\ 949830657@qq.com
}

\begin{abstract}
This paper is carried out deeper analysis aiming at the sustainable development problem that the countries concerned, and create the effective evaluation system. We divide sustainable development capabilities into resource, environment, economy and society four categories; respectively discuss the four aspects of sustainable development capability for each country. In the process of analysis, we start from objective and subjective two aspects, the entropy weight method and the analytic hierarchy process (AHP) are respectively used to analyze, and at last, a perfect comprehensive analysis result was obtained.
\end{abstract}

Keywords: Sustainability; entropy weight method; AHP.

\section{Background}

Sustainable development is one development that not only meets the contemporary needs, but also does not damage the capability that future generations meet need. It is embodied in resource, environment, economy and society these aspects of sustainable development. For a country, whether can maintain the sustainable development determines the development potential and future of the country, so the comprehensive evaluation of the sustainable development capability provides important guiding significance for the country's decision. Nowadays, the world does not have a complete evaluation system on the sustainable development capability, so we have to find an analysis method that can fully reflect the sustainable development capability as soon as possible.

Data used in this article [1] [2] [3] are from national statistical records from 2012 to 2014, the analyzed countries select the United States, China, India, Japan, Germany, Brazil and South Africa seven more typical countries.

\section{Analysis Method}

\subsection{Index Selection.}

In order to measure the sustainable development capability, we must choose the representative indicators as our judgment basis. According to the previous analysis, we can carry out evaluation on resources, environment, economy and society four items, the four evaluation score are set up $S_{1}, S_{2}, S_{3}, S_{4}$, Each item including the corresponding index, it can be done the evaluation of this item, these indicators are set up $r_{i j}(i=1,2,3,4)$. In subsequent analysis, we use the indicators of the corresponding item as an evaluation, the process of comprehensive evaluation results are obtained by the item scores is the second evaluation. In the analysis of the resources, whereas the resource type and quantity of different countries are different, in order to uniformly analyze the sustainable development capability, we only consider from the angle of energy use. Thus evaluation system is established and shown in Table 1.

\subsection{Index Pretreatment.}

In the process of evaluation, in order to make the different dimension and magnitude of indicators can be analyzed together; we need to make standardized processing on every index. For the selected positive indicators, and negative indicators, we need to respectively make standardization on it.

For example, index $r_{31}$ represents the per capita gross national product, and it shows the country's economic strength to some extent, naturally, the higher, the better. For this type of indicators, we use the following formula for standardization: 


$$
\tilde{r}=\frac{r-r_{\min }}{r_{\max }-r_{\min }}
$$

And $r_{\max }$ and $r_{\min }$ respectively are maximum and minimum values that this indicator take in all analyzed countries.

For example, the indicator $r_{11}$ reflects the consumption amount of national non-renewable energy (oil, coal, natural gas), nowadays, increasing shortage of energy, we naturally want to this indicator as small as possible. Similar indicators also have $r_{21}, r_{22}, r_{43}$, for this type of indicator, we use following formula for standardization:

$$
\tilde{r}=\frac{r_{\max }-r}{r_{\max }-r_{\min }}
$$

\begin{tabular}{|c|c|c|}
\hline \multirow{13}{*}{$\begin{array}{c}\text { Sustainable } \\
\text { Development }\end{array}$} & \multirow{3}{*}{ Resources } & Non-renewable energy use per GDP(Except nuclear energy ) \\
\hline & & The proportion of renewable energy generation capacity \\
\hline & & Renewable energy investment per unit of GDP \\
\hline & \multirow{3}{*}{ Environment } & $\mathrm{SO}_{2}$ emission intensities per unit of GDP \\
\hline & & $\mathrm{NO}_{\mathrm{x}}$ emission intensities per unit of GDP \\
\hline & & Forest coverage \\
\hline & \multirow{3}{*}{ Economics } & GDP per capita \\
\hline & & GDP growth rate \\
\hline & & Final consumption expenditure per capita \\
\hline & \multirow{4}{*}{ Society } & Physiological density \\
\hline & & Happiness index \\
\hline & & The aging rate \\
\hline & & Education spend in per unit of GDP \\
\hline
\end{tabular}

Table 1 evaluation system

\subsection{Evaluation Method.}

In order to get a comprehensive evaluation results, we consider from the objective and subjective two aspects to analyze:

Objectively, we analyze the entropy size of each indicator, determine the amount of information that it can provide, obtain weight of each index in one evaluation, then get resources, environment, economy and society these several evaluation results. In the secondary evaluation, we can still use a similar method to get the result of objective evaluation.

Subjectively, we used the analytic hierarchy process to decide the weight of what they should get based on our emphasis degree on resources, environment, economy and society four aspects, make our evaluation system more accord with practice, the result is also easier to understand. For example, in countries where energy resources are scarce, we may more stress sustainability resources, in the secondary evaluation; we can allocate more weight on the resource item. In fact, because the development of each country is different, when using the analytic hierarchy process, we should separately analyze various countries. However, in order to compare the country's sustainable development capability, we adopt a unified standard to analyze it in this thesis.

Entropy weight method. The entropy weight method is used for comprehensive evaluation, the most important thing is to determine the information entropy of each indicator, and the weight of each index is calculated based on it. Determination of $j$ the index entropy in the certain item, steps are as follows:

(1) Calculate the proportion $p_{j k}$ of indicator of $k$ country in $j$ index

$$
p_{j k}=r_{j k} / \sum_{k}^{m} r_{j k}
$$

(2) Calculate the entropy of No. j indicator 


$$
e_{j}=-f \sum_{k=1}^{m} p_{j k} \square \ln p_{j k} \quad f=\frac{1}{\ln m}
$$

When $p_{j k}=0, e_{j}=0$

(3) Calculate entropy $w_{j}$ of No. $\mathrm{j}$ indicators

$$
w_{j}=\left(1-e_{j}\right) / \sum_{j=1}^{n}\left(1-e_{j}\right)
$$

$m$ in the formula is the number of countries, $n$ is the number of index that the item included.

It is important to note that in an evaluation process, the use of the entropy weight method should be limited to index belong to the same items. Therefore, during first evaluation we need to use four times entropy weight method to get relationship between each index and the affiliated index. Thus you can get first evaluation result:

$$
S=\sum_{j=1}^{n}\left(r_{j k} \llbracket w_{j}\right)
$$

Using this method, we can get score of each item of various countries in one evaluation. In the process of comprehensive evaluation, we can use similar approach to get weight that each item gets, and comprehensive evaluation assessment results are obtained. The weight of first evaluation result and the item as shown in Table 2, in the secondary evaluation, entropy of resources, environment, economy and society these four items is $w_{1}=\left(\begin{array}{llll}0.0890 & 0.4779 & 0.3193 & 0.1138\end{array}\right)$

Analytic hierarchy process. The analytic hierarchy process (AHP) is a structured technique for organizing and analyzing complex decisions, based on mathematics and psychology. It simplifies problem by establishing a hierarchy structure model, thus easily deal with the problems. In the previous analysis, we have divided the evaluation system into three layers, and through the entropy weight method to determine the relations between the various items and index. So here we only use analytic hierarchy process to determine the weight that resources, environment, economy and society should gain in the comprehensive evaluation. According to the difference of these four importance, we construct the following judgment matrix:

Table 2 The weight of first evaluation result and the item

\begin{tabular}{|c|c|c|c|c|}
\hline & Resources & Environment & Economics & Society \\
\hline Resources & 1 & 3 & $1 / 2$ & $1 / 4$ \\
\hline Environment & $1 / 3$ & 1 & $1 / 4$ & $1 / 3$ \\
\hline Economics & 2 & 4 & 1 & 1 \\
\hline Society & 4 & 3 & 1 & 1 \\
\hline
\end{tabular}

Can get the greatest eigenvalue of judgment matrix is: $\lambda=4.1707$ and the weight vector $w_{2}=\left(\begin{array}{llll}0.1680 & 0.0870 & 0.3426 & 0.4024\end{array}\right)$.

The judgment matrix are made consistency test:

(1) Calculate the consistency index CI

$C I=\frac{\lambda_{\max }-4}{4-1}=0.0569$

(2) Calculate the consistency ratio

$$
C R=\frac{C I}{R I}=0.063<0.1 \quad R I=0.90 \text { when } n=4
$$

The consistency of judgment matrix is acceptable, the weight vector take $w_{2}$

\section{Analysis Results}

According to the analysis method in 2.3.1 and 2.3.2, we can get score of each item in first evaluation and weight determined by entropy method and the analytic hierarchy process. In the 
secondary evaluation, we hope that we can combine these two methods, present a more comprehensive evaluation results. Here we introduce the weight correction formula:

$W_{i}=\mu w_{1 i}+(1-\mu) w_{2 i}$

In which, $W_{i}$ is weight of No. $\mathrm{i}$ item, $0<\mu<1$ is the weighted coefficient.

So we can get weight by comprehensive analysis of the two methods. According to the actual needs, we can select the appropriate $\mu$ value. In the analysis of this article, we take $\mu=0.5$, and get the weights

$$
W=\left(\begin{array}{llll}
0.1285 & 0.2824 & 0.3310 & 0.2581
\end{array}\right)
$$

Get the comprehensive evaluation result:

$$
S_{\text {fin }}=\sum_{i=1}^{4}\left(W_{i} \sqsubset S_{i}\right)
$$

The first evaluation results and secondary evaluation results are shown in Table 3:

Table 3 The first evaluation results and secondary evaluation results

\begin{tabular}{|c|c|c|c|c|c|}
\hline & $S_{1}$ & $S_{2}$ & $S_{3}$ & $S_{4}$ & $S_{\text {fin }}$ \\
\hline United States & 0.2833 & 0.6804 & 0.8564 & 0.8565 & 0.7331 \\
\hline China & 0.3456 & 0.1546 & 0.2808 & 0.4917 & 0.3079 \\
\hline India & 0.1425 & 0.1217 & 0.1590 & 0.3650 & 0.1995 \\
\hline Japan & 0.4015 & 0.9518 & 0.5084 & 0.2478 & 0.5526 \\
\hline Germany & 0.3995 & 0.7527 & 0.6412 & 0.6240 & 0.6372 \\
\hline Brazil & 0.5666 & 0.9149 & 0.1344 & 0.9383 & 0.6179 \\
\hline South Africa & 0.4558 & 0.0139 & 0.1515 & 0.6358 & 0.2767 \\
\hline
\end{tabular}

\section{Conclusion}

Taken together, the sustainable development capability of United States, Japan, Germany and Brazil these four countries are strong, the national development will remain considerable vitality in the future. From the each item: in terms of resources, the United States, China, India's evaluation is lower, this is because the energy in these countries mainly relying on coal, oil and natural gas, and its sustainable development ability are naturally low, it also means that the energy development in these countries need to transform as soon as possible. In terms of environment, China, India and South Africa's score is low, this and the excess emissions is inseparable, these countries must make corresponding measures to reduce the gap with other countries. The economic aspect, China, India, Brazil and South Africa's score is low, it is because these countries GDP per capita is low, people's consumption rate is low, it is not conducive to the development of national economy. Social aspects, we found that China, India and Japan's score is low, this is mainly because the population physiology density of these countries are higher, it is not conducive to the long-term development of society, in particular, for China and Japan, the aging problems exist, it makes the same score not high; For India, the education investment is low, the investment of education aspect should be increased.

\section{References}

[1] Household final consumption expenditure per capita (constant 2000 US\$) | Data | Table". Data.worldbank.org.

[2] Chart of GDP per capita at current US\$ prices by Google, World Bank data

[3] The Happy Planet Index. http://www.happyplanetindex.org/ 